\title{
Stress induction of HSP3O, the plasma membrane heat shock protein gene of Saccharomyces cerevisiae, appears not to use known stress-regulated transcription factors
}

\author{
Ian J. Seymour $†$ and Peter W. Piper \\ Author for correspondence: Peter W. Piper. Tel: +44 171504 2212. Fax: +44 1713807193. \\ e-mail: piper@bsm.bioc.ucl.ac.uk
}

Department of Biochemistry and Molecular Biology, University College London, London WC1E 6BT, UK

\begin{abstract}
More than one transcription factor contributes to the Saccharomyces cerevisiae heat shock response. Many genes are induced through the activation of heat shock factor (Hsf1), a protein that is constitutively bound to heat shock promoter elements (HSEs). Other genes are switched on by Msn2/Msn4-dependent activation of a quite separate promoter element (the stress response element, STRE). While Hsf1 directs gene activation mainly in response to heat stress, STRE-directed transcription is stimulated not only by heat but also by several other stresses, starvation included. HSP3O, encoding the plasma membrane heat shock protein, is shown in this study to be activated by several stresses. It is most strongly induced with heat shock, ethanol and weak organic acid exposure. The HSP30 promoter has no good agreement to the HSE consensus and its stress activation is unaffected by a mutation (hsf1-m3) that causes defective heat shock activation of Hsf1dependent genes. Activation of HSP3O occurs with some, but not all, STREinducing stresses and is largely unaffected either by loss of the Msn2/Msn4 transcription factors or with mutation of all STRE-like consensus sequences of the promoter. Stress activation of HSP3O appears therefore to involve as yet unidentified components of the yeast transcriptional apparatus.
\end{abstract}

Keywords: yeast, heat shock gene, stress activation, stress response

\section{INTRODUCTION}

Two quite distinct promoter sequences can direct heat shock activation of a Saccharomyces cerevisiae gene. These are the Heat Shock Element (HSE) and the general Stress Responsive Element (STRE) (reviewed by Ruis \& Schüller, 1995; Mager \& De Kruijff, 1995; Piper, 1997; Morano et al., 1998). HSEs are the binding sites for heat shock factor (Hsf1), a transcriptional transactivator that directs gene induction in response to heat stress and chemical agents such as arsenite that cause the intracellular accumulation of abnormal proteins. Recently it was demonstrated that it is a depletion of the 'free'

†Present address: Campden and Chorleywood Food Research Association, Chipping Campden GL55 6LD, UK.

Abbreviations: HSE, heat shock element; PKA, protein kinase A; PDSE, post-diauxic shift element; STRE, stress response element.
Hsp70 chaperone pool in the cells of higher eukaryotes that acts as the signal for $\mathrm{Hsf} 1$ activation, such depletion occurring as Hsp70 associates with the partially heatdenatured proteins in heat shocked cells (Morimoto et al., 1996).

Hsf1-independent but stress-activated yeast promoter elements were originally identified in the CTT1 and DDR2 genes (Belazzi et al., 1991; Marchler et al., 1993; Kobayashi \& McEntee, 1993; Schüller et al., 1994). Activation of these STRE sequences (consensus AGGGG or CCCCT) requires the Msn2 and Msn4 transactivators, proteins that bind STRE sequences in vitro (Martinez-Pastor et al., 1996). STREs, in contrast to HSEs, are activated not just by heat shock but also by a diverse range of other stress conditions including osmostress, oxidative stress, nitrogen starvation and exposure to weak organic acids, low external $\mathrm{pH}$ or ethanol (Marchler et al., 1993; Schüller et al., 1994; Ruis \& Schüller, 1995; Martinez-Pastor et al., 1996). STREs 
also differ from HSEs in that their basal activity and heat induction are very strongly influenced by the levels of protein kinase A (PKA) activity (Marchler et al., 1993).

HSE-mediated and STRE-mediated gene inductions probably serve different functions. Hsf1/HSE-mediated expression appears to primarily direct gene expression that increases the capacity for growth at high temperatures $\left(37-39^{\circ} \mathrm{C}\right)$, not resistance to more severe stress (Ruis \& Schüller, 1995). Thus the bsf1-m3 mutation, which causes loss of the heat-inducibility of HSE sequences and loss of high temperature growth, remarkably has no effect on the acquisition of tolerance to extreme temperatures $\left(50^{\circ} \mathrm{C}\right)$. In addition, tolerance to extreme temperatures can be induced by treatments which cause no HSE induction, such as osmotic dehydration or exposure to weak organic acids (Piper, 1993). In contrast, STRE-mediated transcription has all the properties of a system, activated by diverse types of sublethal stress, whereby cells are reprogrammed for the survival of more severe stress, such as desiccation, prolonged starvation, freezing, etc. (Ruis \& Schüller, 1995). Consistent with this the msn $2 m s n 4$ mutant that is defective in the transcription activators needed for STRE action exhibits hypersensitivity to several stresses (Martinez-Pastor et al., 1996). There appears to be a good correlation between tolerances to these extreme stresses in S. cerevisiae and the levels of STRE-regulated gene products (Ruis \& Schüller, 1995; Mager \& De Kruijff, 1995; Piper, 1997). In general, the expression of STRE-regulated genes is minimal in cells in rapid glucose growth, being activated under growth-limiting conditions through a number of signal transduction pathways. The high-osmolarity-activated HOG (high osmolarity glycerol) pathway is one stress sensing pathway that influences STRE activity (Schüller et al., 1994). Another very important influence is PKA activity, this being inversely related to the levels of several stress tolerances in yeast (Belazzi et al., 1991; Marchler et al., 1993; Schüller et al., 1994) including thermotolerance (Piper, 1993). A lowering of PKA activity slows growth, increases basal STRE activity and increases the responsiveness of STRE sequences to heat stress (Marchler et al., 1993).

It appears that an individual yeast heat shock gene can be under HSE control, under STRE control, or controlled by both the HSE and STRE. In addition it may be subject to other influences (e.g. UBI4 which has an element causing higher expression levels in respiratory as compared to fermentative cultures; Watt \& Piper, 1997). Studies on different yeast heat shock gene promoters have indicated that the heat induction of a few is regulated by the HSE alone. These promoters include SSA4, encoding a form of Hsp70 (Boorstein \& Craig, 1990a), and HSP82, encoding the heat-inducible Hsp90 (McDaniel et al., 1989). Others are under the control of STRE (not HSE) sequences; sometimes multiple synergistically acting STREs as in HSP12 (Varela et al., 1995). Heat shock genes of yet a third category (e.g. SSA3; Boorstein \& Craig, 1990b) seem to be regulated by both the HSE- and PKA-responsive sequences. In these latter promoters HSEs and STREs may operate independently although SSA3 contains a STRE-like element [the post-diauxic shift element (PDSE)] that seems to be dependent on a downstream HSE for its activity (Boorstein \& Craig, 1990b). Whether a heat shock gene is under HSE or STRE control in turn influences its stress induction pattern. Thus STREregulated genes (e.g. HSP12) are induced by several stresses and low PKA activity. In contrast the HSEregulated SSA4 appears to be induced primarily by heat shock alone. However, not all STRE-like promoter sequences are identical, since the different STREs controlling the genes for subunits of the trehalose synthase complex do not all direct the same patterns of regulation (Winderickx et al., 1996).

In this study we attempted to identify whether HSP30, the gene encoding the integral plasma membrane heat shock protein of S. cerevisiae (Panaretou \& Piper, 1992; Régnacq \& Boucherie, 1992; Piper et al., 1994, 1997), is under HSE or STRE control. To our surprise we obtained data that indicate HSP3O is not under the control of either of these elements, suggesting that a third system for the activation of genes by heat shock must exist in yeast.

\section{METHODS}

Yeast strains, yeast culture and stress exposure. The yeast strains used for this study are listed in Table 1. Yeast growth, media recipes, DNA transformation procedures, stress exposure protocols, RNA extraction and the probing of Northern blots were exactly as reported previously (Watt \& Piper, 1997; Piper et al., 1997, 1998). The starvation of cultures for nitrogen was according to the procedure of Marchler et al. (1993).

Site-directed mutagenesis of HSP3O promoter sequences. Plasmid YIp30 was constructed by ligation of the $2.4 \mathrm{~kb}$ NdeI fragment of pUC19HSP30 (Régnacq \& Boucherie, 1992) into NdeI-cleaved YIplac204 (Geitz \& Sugino, 1988). YIp30 contains the entire HSP30 ORF and 963 bp of upstream DNA, together with a unique EcoRV site within the TRP1 gene of the vector. Mutagenesis of the HSP30 promoter of YIp30 employed the oligonucleotide primers shown in Table 2, each consensus sequence being altered in turn by repeated use of the Stratagene QuickChange site-directed mutagenesis kit. After mutant promoter sequences had been confirmed by DNA sequence analysis, the YIp30-derived plasmids bearing multiple promoter mutations were digested (100 ng) with EcoRV then used to transform strain KT3 (Table 1) to tryptophan prototrophy. Correct integration of promoter mutant $h s p 30$ alleles at the $\operatorname{trp} 1$ locus was confirmed by Southern blotting, $T R P 1^{+}$transformants that were genomic insertions of single copies of the plasmid being used for further studies. KT3 transformed with YIp30 derivatives are designated by a suffix to the strain name (e.g. KT3-YIp30 is KT3 transformed with unmutated YIp30; KT3-STRE1(M) is KT3 transformed with YIp30 containing the STRE1(M) mutation in Table 2; KT3STRE1(M)-PDSE1-6(M) is KT3 transformed with YIp30 containing all the mutations in Table 2; while KT3-PDSE1$6(\mathrm{M})$ has all six of the PDSE consensus sequences mutated as in Table 2). 
Table 1. Yeast strains used in this study

\begin{tabular}{|c|c|c|}
\hline Strain & Genotype & Reference \\
\hline BJ2168 & a leu2-3,112 trp1 ura3-52 prb1-1122 pep4-3 prc407 gal2 & Piper et al. (1997) \\
\hline W303-1A & a ade 2 can 1 bis 3 leu 2 trp 1 ura 3 & Martinez-Pastor et al. (1996) \\
\hline$m s n 2 m s n 4$ & W303-1A msn2- $33:: H I S 3 m s n 4-1::$ TRP1 & Martinez-Pastor et al. (1996) \\
\hline MYY290 & a leu 2 bis 3 ura 3 & Smith \& Yaffe (1991) \\
\hline JC302-26B & $\mathrm{JC} 482$ ras $2::$ LEU2 & Belazzi et al. (1991) \\
\hline JC303-79 & $\mathrm{JC} 482$ ras $2:$ : LEU2 sra1.13 & Belazzi et al. (1991) \\
\hline
\end{tabular}

Table 2. Primers used for site-directed mutagenesis of the HSP30 gene promoter within plasmid YIp30

\begin{tabular}{|llc|}
\hline Primer & \multicolumn{1}{c|}{ Sequence* $^{*}$} & Location $\dagger$ \\
\hline STRE1-M & GCCGTATTCTTCTTGCGCGTAAGAAAGTTG & -803 to -774 \\
PDSE1-M & GCAGGGTCCGAATCGCTTGGAAAACCGC & -675 to -647 \\
PDSE2-M & CCTTGCGTCAGCGAGCGCGATTTTGTTGG & -600 to -572 \\
PDSE3-M & CGACTCTTATTCCGAAGCGATCCGCCACATCC & -430 to -399 \\
PDSE4-M & CTTCGTTATTTCATCAGCGCTCTCTGCTATGGCAAGG & -366 to -330 \\
PDSE5-M & GCATCTCATCGAAAACTTTCCTCAGCGAGATTGGCCAAAG & -314 to -275 \\
PDSE6-M & GTTAGAAAGTATAATATCGGTCGCTCGCCTCATTACAAGTTG & -256 to -215 \\
\hline
\end{tabular}

*Each of these primers was used, together with a primer of exactly complementary sequence (not listed), in the QuickChange procedure for site-directed mutagenesis. Nucleotide changes from the wild-type HSP30 promoter sequence are underlined and in bold.

† Annealing position relative to the A of the ATG start codon as +1 .

\section{RESULTS}

\section{Stresses that lead to strong induction of $\mathrm{Hsp30}$ mRNA}

Previous work has shown that Hsp30 mRNA is induced during entry to stationary phase under conditions where glucose becomes limiting (Régnacq \& Boucherie, 1992; Riou et al., 1997); also that Hsp30 protein is induced in purified plasma membranes by glucose limitation, heat shock and exposure to either ethanol or sorbic acid (Panaretou \& Piper, 1992; Régnacq \& Boucherie, 1992; Piper et al., 1994, 1997). The effects of different stresses on Hsp30 mRNA levels are shown in Fig. 1. HSP30 mRNA is detectable even during exponential growth on glucose (Fig. 1, lane 1), but is further induced by heat shock and ethanol (12- and 7-fold increases over basal levels, respectively, in this experiment). Different kinds of severe osmostress ( $2 \mathrm{M}$ sorbitol, $1 \mathrm{M} \mathrm{NaCl}$ or $1 \mathrm{M}$ $\mathrm{KCl}$ for $1 \mathrm{~h}$ ) cause only a small induction (two- to threefold). Moderate induction is also seen with oxidative stress $(0.4 \mathrm{mM}$ hydrogen peroxide, $1 \mathrm{mM}$ menadione or $1 \mathrm{mM}$ cadmium chloride for $1 \mathrm{~h}$; Fig. 1 ). Cold shock or more moderate osmostresses $(0.5 \mathrm{M}$ $\mathrm{NaCl}$ or $0.5 \mathrm{M} \mathrm{KCl}$ for $1 \mathrm{~h}$ ) do not cause induction (Fig. 1). In other experiments (see below) we found that weak organic acid stress was an even stronger inducer of HSP30 than heat or ethanol treatment.

Both actin (ACT1) and PDA1 transcript levels were used to control for RNA loading in Fig. 1. The latter mRNA has been suggested as a superior loading control for Northern blots compared to actin mRNA, since the levels of actin mRNA are not constant in cells (Wenzel et al., 1995). In general we have found PDA1 mRNA to be a satisfactory loading control, although its levels increase slightly under conditions of severe osmostress (Fig. 1).

Since heat, ethanol and weak acid exposure are the strongest inducers of Hsp30 mRNA, these stress regimes and carbon or nitrogen starvation were used in most subsequent experiments.

\section{HSP30 appears not to be subject to Hsf1/HSE regulation}

Several heat shock genes are activated by Hsf1 bound to HSE sequences (see Introduction). The HSE usually comprises at least three alternating adjacent repeats of the 5 bp motif nGAAn, each repeat comprising one halfturn of the DNA double helix (Sorger, 1991). However, 


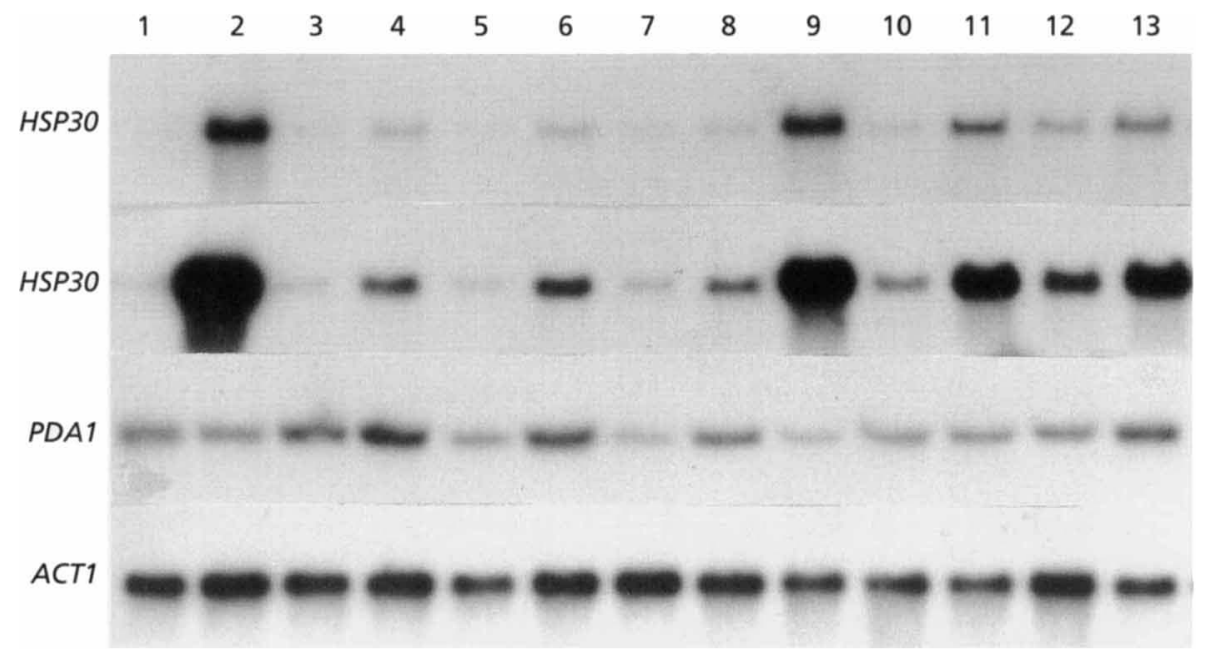

Fig. 1. Northern analysis of RNA from cells of strain BJ2168 subjected to different stresses. Hybridization was to radiolabelled probes specific for HSP30, PDA1 or ACT1 probes (the latter two being loading controls). RNA samples (each $20 \mu \mathrm{g}$ ) were from unstressed $30^{\circ} \mathrm{C}$ control cells (lane 1) or from cells subjected to heat shock for $1 \mathrm{~h}$ at $39^{\circ} \mathrm{C}$ (lane 2); cold shock for $3 \mathrm{~h}$ at $15^{\circ} \mathrm{C}$ (lane 3); osmostress [either $2 \mathrm{M}$ sorbitol (lane 4), $0.5 \mathrm{M} \mathrm{NaCl}$ (lane 5), $1 \mathrm{M} \mathrm{NaCl}($ lane 6$), 0.5 \mathrm{M} \mathrm{KCl}$ (lane 7) or $1 \mathrm{M} \mathrm{KCl}$ (lane 8)]; $6 \%(\mathrm{w} / \mathrm{v}$ ) ethanol (lane 9); $0.4 \mathrm{mM}$ hydrogen peroxide (lane 10); $1 \mathrm{mM}$ menadione (lane 11); $1 \mathrm{mM}$ cadmium chloride (lane 12) and $10 \mu \mathrm{M}$ 4-nitroquinoline- $N$-oxide (lane 13). For samples 4-13 stress exposure was for $1 \mathrm{~h}$ at $30^{\circ} \mathrm{C}$. Both short ( $10 \mathrm{~min}$; first row) and long ( $2 \mathrm{~h}$; second row) exposures of the HSP3O blot are shown.

STREI

Fig. 2. Nucleotide sequence of the HSP3O 5'-noncoding region. The $4 / 5 \mathrm{bp}$ agreements to STRE and PDSE consensus sequences are shown in bold, their orientation being indicated by an underlying arrow. The two closest agreements to the HSE consensus are indicated by dotted overlining and the two TATA sequences are boxed. in at least one situation (the CUP1 promoter) two alternating nGAAn repeats are sufficient for Hsf1 binding and in this instance Hsf1 interaction provides both heat and oxidant (menadione) inducibility (Liu \& Thiele, 1996). Examination of the promoter region of HSP30 (Fig. 2) did not reveal two or more juxtaposed nGAAn repeats that probably could constitute a functional HSE. In addition, heat induction of HSP30 was unaffected by a mutation ( $h s f 1-m 3$ ) that abolishes the heat inducibility of HSE sequences (see Fig. 5). hsf1-m3 is a nonsense mutation within the HSF1 gene that probably causes a considerable reduction in Hsf1 levels, since the production of functional Hsf1 in this mutant requires nonsense suppression during the translation of Hsf1 mRNA (Lindquist \& Kim, 1996). This mutation, although allowing the basal HSE activity essential for yeast viability, results in a complete absence of the heatinducibility of HSE sequences (Smith \& Yaffe, 1991). From the apparent lack of a HSE in the HSP30 promoter and absence of any influence of $h s f 1-m 3$ we concluded that HSP30 is unlikely to be subject to Hsf1/HSE regulation.

\section{Mutagenesis of putative STRE and PDSE-like elements within the HSP30 gene promoter}

Further examination of the HSP 30 promoter (Fig. 2) also did not reveal any perfect match to the STRE consensus AGGGG (or CCCCT), even though this is a gene clearly induced by a number of different stresses (Fig. 1). There were, however, a number of $4 / 5$ bp agreements to the STRE consensus, these being a CCCCG sequence at 


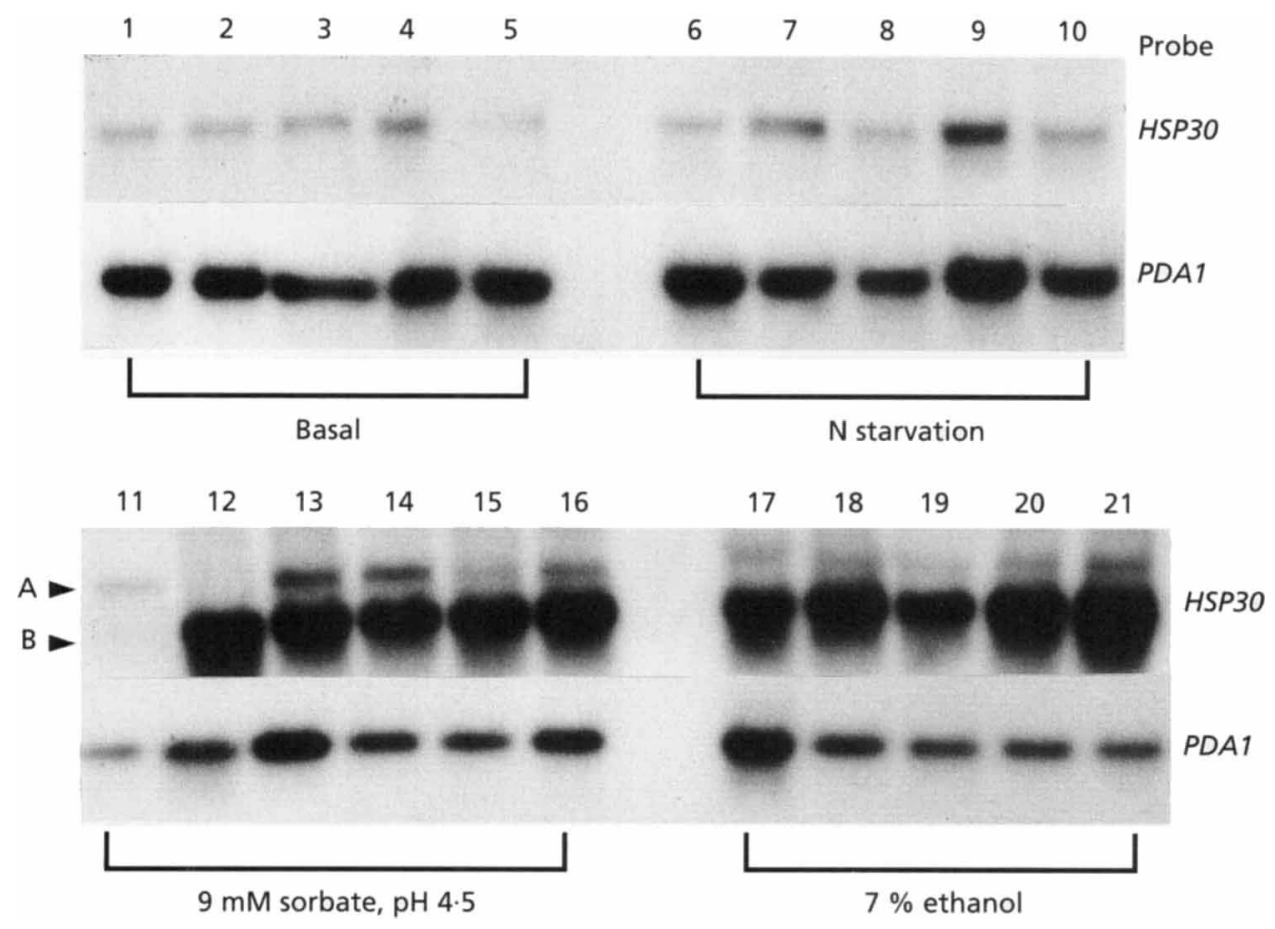

Fig. 3. Analysis of Hsp30 mRNA in wild-type BJ2168 (lanes 1, 6, 12 and 17) and untransformed KT3 cells (lane 11); also transformants KT3-YIp30 (lanes 2, 7, 13 and 18), KT3-STRE1(M) (lanes 3, 8, 14 and 19), KT3-STRE1(M)-PDSE1-6(M) (lanes 4, 9, 15 and 20) and KT3-PDSE1-6(M) (lanes 5, 10, 16 and 21). The RNA samples analysed were from either unstressed cells (lanes 1-5) or cells exposed for $1 \mathrm{~h}$ at $30^{\circ} \mathrm{C}$ to nitrogen starvation (lanes $6-10$ ), weak acid (sorbate) stress at pH 4.5 (lanes 11-16) or $7 \%$ ethanol (lanes 17-21). In KT3 transformants the normal 1.3 kb Hsp30 mRNA (B) originates from the HSP30 gene introduced on the vector, since this RNA is absent in untransformed KT3 cells which synthesize only a larger transcript (A) originating from the hsp30:: URA3 allele (lane 11). The latter transcript is not visible in uninduced cell samples (lanes 1-10). The blots were also probed with PDA1 mRNA as a control for RNA loading.

-785 indicated as 'STRE1' in Fig. 2, as well as several AGGGA or TCCCT sequences. The latter are designated PDSE1-6 in Fig. 2 since they more closely correspond to the consensus of the PDSE or 'postdiauxic shift' element identified in the SSA3 promoter by Boorstein \& Craig (1990b). This SSA3 PDSE element contains a similar motif $(4 / 5 \mathrm{bp}$ match) to the STRE consensus and it, like the STRE, is activated by low PKA activity levels. However, the PDSE appears not to be identical to the STRE since it is not under Msn2 Msn4 transcription factor control (Ruis \& Schüller, 1995) and, at least in the SSA3 promoter, appears to require a downstream HSE to cause heat activation (Boorstein \& Craig, 1990b).

PDR12, another gene strongly induced by weak organic acid stress (Piper et al., 1998), also possesses several AGGGA sequences within its promoter (not shown). We therefore wondered if this might constitute a stress responsive element in PDR12 and HSP30. At least two key residues within each of these $4 / 5$ bp agreements to the STRE and PDSE consensus sequences of the HSP30 promoter (Fig. 2) were therefore mutated on a HSP30 gene present within the integrative vector YIp30 (see Methods). These mutations (Table 2) were introduced into YIp30 singly and in combination. After confirmation of the introduced changes by sequencing, the plasmids bearing these promoter mutations were integrated into the defective trp1 allele of the hsp30::URA3 yeast strain KT3 (Table 1) by transformation to tryptophan prototropy. KT3 does not synthesize a normal $1.3 \mathrm{~kb}$ Hsp30 mRNA since it has a $U R A 3$ gene fragment insertional inactivation of the HSP30 gene, at +18 within the HSP30 ORF (Piper et al., 1997). Thus normal-sized Hsp30 mRNA in KT3 transformed with YIp30-derived plasmids originates solely from the introduced HSP3O allele (Fig. 3).

Exponential phase YEPD cultures of wild-type BJ2168, also transformants KT3-YIp30, KT3-STRE1(M), KT3STRE1(M)-PDSE1-6(M) and KT3-PDSE1-6(M) (see Methods), were transferred to fresh YEPD and either placed for $1 \mathrm{~h}$ at $30^{\circ} \mathrm{C}$ (nonstressed control cells), heat shocked for $1 \mathrm{~h}$ at $39^{\circ} \mathrm{C}$, or incubated for $1 \mathrm{~h}$ at $30^{\circ} \mathrm{C}$ with either $7 \%$ ethanol at $\mathrm{pH} 7$ or $9 \mathrm{mM}$ sorbic acid at $\mathrm{pH} 4.5$. An identical set of cell samples was also incubated in nitrogen starvation medium. RNA was then prepared from these cultures and analysed by Northern blotting (Fig. 3). Remarkably, mutation of all the STRE and PDSE consensus sequences of the promoter [as in KT3-STRE1(M)-PDSE1-6(M)] had no dramatic effects on stress induction, although some minor effects cannot be ruled out. It caused less than twofold changes in HSP30 induction in response to 


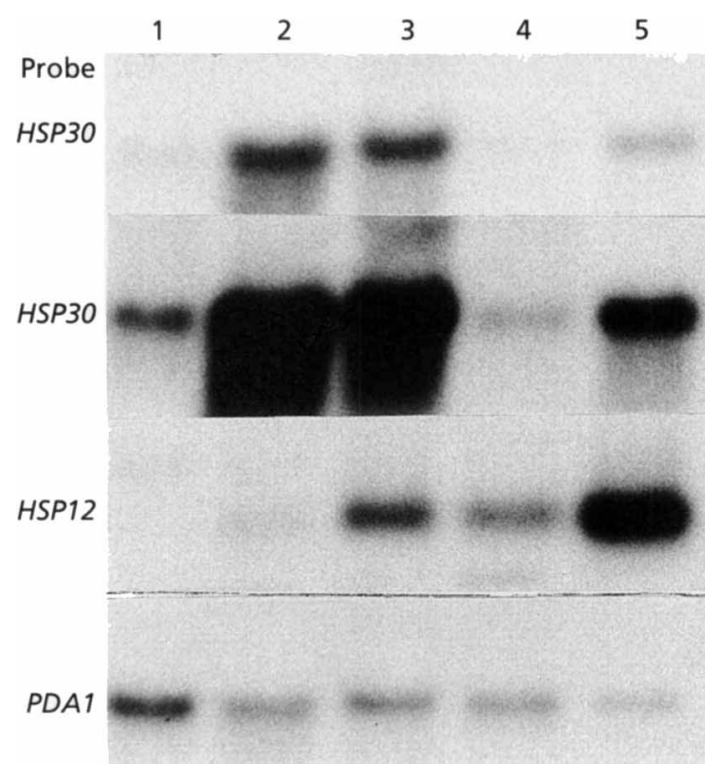

Fig. 4. Comparison of the stress-induced expression of HSP3O and HSP12. RNA samples $(10 \mu \mathrm{g})$ analysed were from either unstressed BJ2168 cells (lane 1) or cells stressed with either $9 \mathrm{mM}$ sorbic acid $\left(1 \mathrm{~h}\right.$ at $30^{\circ} \mathrm{C}$ at $\mathrm{pH} 4.5$ ) (lane 2), $7 \%$ ethanol $\left(1 \mathrm{~h}\right.$ at $30^{\circ} \mathrm{C}$ at $\mathrm{pH} \mathrm{6.5)} \mathrm{(lane} \mathrm{3),} \mathrm{nitrogen} \mathrm{starvation}\left(1 \mathrm{~h}\right.$ at $30^{\circ} \mathrm{C}$ in nitrogen starvation medium) (lane 4 ) or heat shock ( $1 \mathrm{~h}$ at $39{ }^{\circ} \mathrm{C}$ ) (lane 5). Both short (first row) and long (second row) exposures of the HSP3O blot are shown.

either sorbate at $\mathrm{pH} 4.5$ (Fig. 3, lanes 11-16), $7 \%$ ethanol (Fig. 3, lanes $17-21$ ) or a $39^{\circ} \mathrm{C}$ heat shock (data not shown). Sorbate, ethanol and heat shock are all STRE-inducing stresses (Marchler et al., 1993; Martinez-Pastor et al., 1996). Furthermore the wild-type and promoter mutant HSP3O alleles were not induced by nitrogen starvation (Fig. 3, lanes 1-10). Indeed while performing this analysis we noticed that while HSP3O is induced by glucose starvation, it is not induced by nitrogen starvation. The latter condition strongly induces STRE controlled genes (Marchler et al., 1993). Since mutation of two nucleotides within each of the closest agreements to the STRE and PDSE did not significantly affect the stress induction of HSP30, we concluded that the strong sorbate, ethanol and heat shock inductions of this gene are largely independent of STRE- or PDSE-like promoter sequences. This conclusion is reinforced by studies of these inductions in transcription factor mutants, described below.

\section{Comparison of stress-induced expression of HSP3O and HSP12}

The data in Fig. 3 indicate that the stress induction of HSP3O is not due to the STRE-like sequences within the promoter. Moreover HSP3O is not appreciably induced by nitrogen starvation (Fig. 3, lanes 6-10), a strong inducer of STRE-regulated genes. To show that our conditions of nitrogen starvation would indeed cause STRE induction, we also measured Hsp12 mRNA levels. HSP12 is a good example of a STRE-regulated gene, its promoter having five repeats of the AGGGG STRE motif that confer activation in response to osmostress, heat shock and transition to stationary phase (Varela et al., 1995). Northern analysis of the changes to HSP30 and HSP12 transcripts in strain BJ2168 in response to weak acid stress, ethanol, nitrogen starvation and heat shock are shown in Fig. 4. Transcripts for both Hsp30 and Hsp12 are at low levels during exponential growth and appreciably induced in response to ethanol (Fig. 4, lanes 1 and 3). Hsp30 mRNA is strongly induced, and Hsp12 mRNA is moderately induced, by weak organic acid stress (Fig. 4, lane 2). Conversely, Hsp30 mRNA is less strongly induced by heat shock than Hsp12 mRNA. Most noteworthy, however, is the finding that Hsp30 mRNA is not induced under the conditions of nitrogen starvation that induce Hsp12 mRNA (Fig. 4, lane 4). Thus although these two heat shock genes share some similarities in their stress activations, there are also major differences that show that HSP12 and HSP3O are not subject to identical controls. HSP12 is induced by both nitrogen starvation and moderate osmostress (Varela et al., 1995), stresses that do not induce HSP3O (Figs 1, 3 and 4). In addition there are differences in the magnitudes of the inductions of these two genes by sorbate and by heat (Fig. 4).

\section{HSP30 stress induction is largely unaffected by the loss of Msn2 and Msn4, two proteins needed for strong stress activation of the STRE}

The Msn2 and Msn4 zinc finger proteins are transcriptional activators needed for the strong stress activation of several STRE-regulated genes, such as CTT1, DDR2 and HSP12 (Martinez-Pastor et al., 1996). STRE activation is severely reduced in $m s n 2 m s n 4$ mutant cells, cells that are also hypersensitive to several stresses. The responses of Hsp30 mRNA and Hsp12 mRNA to two STRE-inducing stresses, heat and sorbate, were studied in isogenic wild-type and $m s n 2 m s n 4$ cells (Fig. 5). The strong sorbate induction of HSP30 was not diminished in $m s n 2 m s n 4$ cells, although the much weaker sorbate induction of HSP12 was lost (Fig. 5, lanes 1-4). However, there did appear to be a slight reduction in the weaker heat induction of HSP 30 in $m s n 2 m s n 4$ mutant cells, this being about $50 \%$ of wildtype (Fig. 5, lanes 1, 2, 5 and 6). These results are a further indication that much of the stress induction of HSP3O is mainly independent of the STRE.

\section{HSP30 stress induction is not appreciably controlled by levels of PKA activity}

Lowering PKA activity increases STRE-mediated gene expression and the responsiveness of STREs to stress (see Introduction). We investigated basal and heatshock-induced expression of HSP3O in glucose-grown cultures of three strains (Belazzi et al., 1991; Table 1) with either normal (JC482), constitutively high (JC30379) or constitutively low (JC302-26B) PKA activity levels. Although low PKA activity causes elevated expression of STRE-regulated genes, it was not 


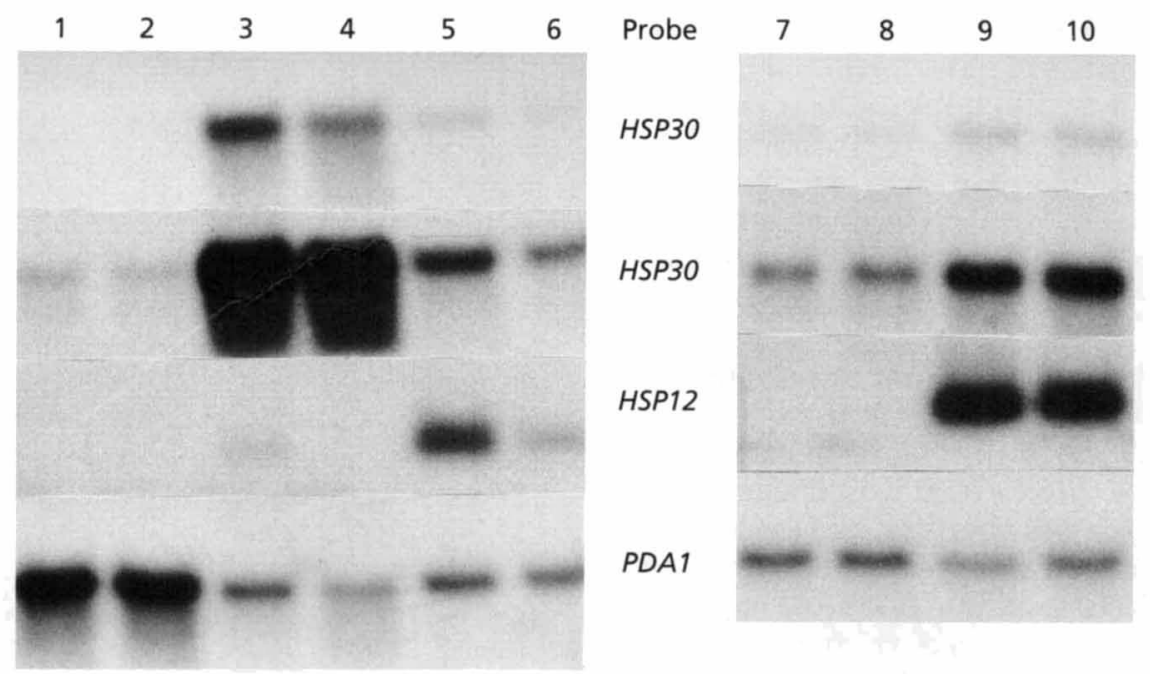

Fig. 5. Comparison of the stress-induced expression of HSP3O and HSP12 in transcription factor mutants. RNA samples $(10 \mu \mathrm{g})$ were from msn2 msn4 cells (lanes 2, 4 and 6) and their isogenic wild-type W303-1A (lanes 1, 3 and 5); also the hsfi-m 3 mutant (lanes 8 and 10) and its isogenic wild-type parent MYY290 (lanes 7 and 9). Cells were either unstressed (lanes 1, 2, 7 and 8), weak-acid-stressed at pH 4.5 with $9 \mathrm{mM}$ sorbate for $1 \mathrm{~h}$ at $30^{\circ} \mathrm{C}$ (lanes 3 and 4) or heat shocked for $1 \mathrm{~h}$ at $39^{\circ} \mathrm{C}$ (lanes 5, 6, 9 and 10). The blots were probed with HSP30, HSP12 and PDA1 sequences. Both short (first row) and long (second row) exposures of the HSP3O blot are shown.

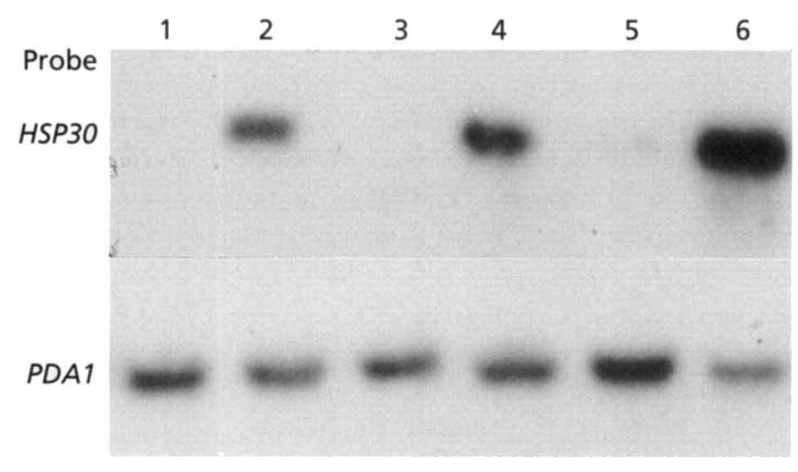

Fig. 6. Basal and heat-induced HSP3O expression in strains with normal, constitutively high or constitutively low PKA activity. RNAs were from strains JC482 (lanes 1 and 2), JC303-79 (lanes 3 and 4) and JC302-26B (lanes 5 and 6), either in exponential growth on glucose (lanes 1,3 and 5 ) or heat shocked for $1 \mathrm{~h}$ at $39^{\circ} \mathrm{C}$ (lanes 2, 4 and 6).

associated with an increased basal level of HSP30 expression (Fig. 6). PKA activity levels also did not cause major changes to the heat shock induction of this gene (Fig. 6).

\section{DISCUSSION}

When we commenced this study we suspected that, since HSP 30 is a heat shock gene, it would be controlled by either the HSE or the STRE, or perhaps be controlled by both of these promoter elements. Unexpectedly our data indicate that stress activation of this gene is due to neither of these promoter elements. HSP30 is induced by more than one stress, including several that also induce the STRE [heat shock, ethanol, sorbate exposure (Figs 1 and 3-6) and carbon starvation (Régnacq \& Boucherie, 1992; Riou et al., 1997)]. However, it is not induced by certain other STRE-inducing stresses (moderate osmostress, nitrogen starvation). Moreover the basal and stress-induced expressions of this gene, unlike those of the STRE and PDSE, appear to be largely independent of PKA activity (Fig. 6). Further evidence that HSP3O is not STRE-regulated was obtained by comparing its stress induction with that of the well-characterized STREregulated gene HSP12 (Figs 4 and 5).

There are other stress responsive promoter elements in yeast besides the HSE and STRE. One is the binding site for Yap1 protein homologues (consensus TTACTAA; Fernandes et al., 1998). Several of the genes responsive to oxidative stress are under Yap1 control. However, the HSP30 promoter does not have a perfect agreement to the Yap1 binding consensus and, while it is slightly induced by hydrogen peroxide (Fig. 1), shows a normal sorbate and ethanol induction in yap1 mutant cells (data not shown). There are, however, a number of Yap1 homologues in yeast which have yet to be assigned a function (Fernandes et al., 1998), as well as possible degenerate Yap1 recognition sequences in the HSP3O promoter. We have therefore not yet excluded the possibility that the stress induction of HSP 30 is due to one of these Yap1-related factors.

It is possibly significant that the strongest stress inducers of HSP 30 (Figs 1 and 3-6) appear to be those treatments that will increase membrane fluidity. Elevated temperatures and ethanol both increase fluidity of the 
phospholipid bilayer. Alkanols and organic acids such as sorbate have propensities to partition into the bilayer which increase as their hydrophobicity increases. It is possible therefore that HSP3O is responding to a membrane-fluidity-sensing system, a system that stimulates a signal transduction pathway acting on this and probably other genes in a HSE- and STRE-independent manner. Part of the function of such a system may be to minimize the effects of stress on the membrane. HSP30 encodes a highly hydrophobic integral membrane protein which, though detectable in exponentially growing yeast cells, increases dramatically in level in response to heat shock, ethanol and sorbate treatment (Panaretou $\&$ Piper, 1992; Régnacq \& Boucherie, 1992; Piper et al., 1994, 1997). There are preliminary indications that this Hsp30 protein may have several actions at the plasma membrane. Besides exerting a negative effect on stressinduced increases in plasma membrane $\mathrm{H}^{+}$-ATPase activity (Piper et al., 1997), it also acts to reduce membrane fluidity at higher growth temperatures (K. Obuchi, unpublished results). These actions appear to be reducing the effects of sublethal stress, since Hsp30 null cells take longer to resume growth after a number of nonlethal stress exposures that cause temporary growth arrest (Piper et al., 1997; K. Obuchi, unpublished results).

The sensor of the heat shock response in higher eukaryotic cells is widely thought to be Hsp70 (see Introduction). However, there is now increasing evidence that membrane composition can affect the sensing of heat stress, at least in microbes. Membrane lipid composition affects HSE activation by heat shock in yeast (Chatterjee et al., 1997), as well as the heat shock responsiveness of the unicellular cyanobacterium Synechocystis PCC 6803 (Horváth et al., 1998). Hsp30 null cells have higher membrane fluidity at high temperatures (K. Obuchi, unpublished results), but whether they display an altered stress response still remains to be determined.

One of the strongest inducers of HSP30 appears to be weak organic acid stress (Figs 3-5). Water-soluble weak organic acid food preservatives such as sorbic acid appear to induce a discrete stress response in yeast that assists adaptation to growth in the presence of these compounds (Piper et al., 1987, 1988). This response leads to strong induction of two plasma membrane proteins, one of which is Hsp30 (Piper et al., 1997) and the other an ATP-binding cassette transporter (Pdr12; Piper et al., 1998). Weak organic acid (sorbate) stress also induces the STRE, but this is not the promoter element by which this stress causes induction of HSP30 (Figs 3-5). Instead an alternative weak-acid-responsive element appears to exist, a putative element that may be especially important in the adaptive response to weak organic acid stress. To identify this element, we are currently constructing deletions of the HSP30 promoter, and also analysing weak-acid-sensitive mutants so as to identify the proteins involved in the sensing of weak acid stress and the signalling of this stress to the transcription apparatus.

\section{ACKNOWLEDGEMENTS}

We are indebted to M. Yaffe and H. Ruis for strains. This project was supported by BBSRC grant 31/D10371 and a BBSRC Studentship (I. J.S.).

\section{REFERENCES}

Belazzi, T., Wagner, A., Wieser, R., Schanz, M., Adam, G., Hartig, A. \& Ruis, H. (1991). Negative regulation of transcription of the Saccharomyces cerevisiae catalase $\mathrm{T}$ (CTT1) gene by cAMP is mediated by a positive control element. EMBO J 10,585-592.

Boorstein, W. R. \& Craig, E. A. (1990a). Structure and regulation of the SSA4 HSP70 gene of Saccharomyces cerevisiae. J Biol Chem 265, 18912-18921.

Boorstein, W. R. \& Craig, E. A. (1990b). Regulation of a yeast HSP70 gene by a cAMP responsive transcriptional control element. EMBO J 9, 2543-2553.

Chatterjee, M. T., Khalawan, S. A. \& Curran, B. P. G. (1997). Alterations in cellular lipids may be responsible for the transient nature of the yeast heat shock response. Microbiology 143, 3063-3068.

Fernandes, L., Rodrigues-Pousada, C. \& Struhl, K. (1998). Yap, a novel family of eight bZIP proteins in Saccharomyces cerevisiae with distinct biological functions. Mol Cell Biol 17, 6982-6993.

Geitz, R. D. \& Sugino, A. (1988). New yeast-Escherichia coli shuttle vectors constructed with in vitro mutagenised yeast genes lacking six-base pair restriction sites. Gene 74, 527-534.

Horváth, I., Glatz, A., Varvasovszki, V. \& 8 other authors (1998). Membrane physical state controls the signalling mechanism of the heat shock response of Synechocystis PCC 6803: identification of $h s p 17$ as a fluidity gene. Proc Natl Acad Sci USA 95, 3513-3518.

Kobayashi, N. \& McEntee, K. (1993). Identification of cis and trans-components of a novel stress regulatory pathway in Saccharomyces cerevisiae. Mol Cell Biol 13, 248-256.

Lindquist, S. \& Kim, G. (1996). Heat-shock protein 104 expression is sufficient for thermotolerance in yeast. Proc Natl Acad Sci USA 93, 5301-5306.

Liu, X.-D. \& Thiele, D. J. (1996). Oxidative stress induces heat shock factor phosphorylation and HSF-dependent activation of yeast metallothionein gene transcription. Genes Dev 10, 592-603.

McDaniel, D., Caplan, A. J., Lee, M.-S., Adams, C. C., Fishel, B. R., Gross, D. S. \& Garrard, W. T. (1989). Basal-level expression of the yeast HSP82 gene requires a heat shock regulatory element. Mol Cell Biol 9, 4789-4798.

Mager, W. H. \& De Kruijff, A. J. J. (1995). Stress-induced transcriptional activation. Microbiol Rev 59, 506-531.

Marchler, G., Schuller, C., Adam, G. \& Ruis, H. (1993). A Saccharomyces cerevisiae UAS element controlled by kinase A activates transcription in response to a variety of stress conditions. EMBO J 12, 1997-2003.

Martinez-Pastor, M. T., Marchler, G., Schuller, C., Marchler-Bauer, A., Ruis, H. \& Estruch, F. (1996). The Saccharomyces cerevisiae zinc finger proteins Msn2 and Msn4 are required for transcriptional induction through the stress-response element (STRE). $E M B O ~ J ~ 15,2227-2235$.

Morano, K., Liu, P. \& Thiele, D. (1998). Protein chaperones and the heat shock response of Saccharomyces cerevisiae. Curr Opin Microbiol 1, 197-203.

Morimoto, R. I., Kroeger, P. E. \& Cotto, J. J. (1996). The transcriptional regulation of heat shock genes: a plethora of heat shock factors and regulatory conditions. In Stress-Inducible Trans- 
criptional Responses, pp. 139-163. Edited by U. Feige, R. I. Morimoto, I. Yahara \& B. S. Polla. Boston, MA : Birkhauser.

Panaretou, B. \& Piper, P. W. (1992). The plasma membrane of yeast acquires a novel heat shock protein ( $\mathrm{Hsp30}$ ) and displays a decline in proton-pumping ATPase levels in response to heat shock and the entry to stationary phase. Eur J Biochem 206, $635-640$.

Piper, P. W. (1993). Molecular events associated with acquisition of heat tolerance by the yeast Saccharomyces cerevisiae. FEMS Microbiol Rev 11, 339-356.

Piper, P. W. (1997). The yeast heat shock response. In Yeast Stress Responses, pp. 75-99. Edited by S. Hohmann \& W. H. Mager. Austin, TX: R. G. Landes.

Piper, P. W., Talreja, K., Panaretou, B., Moradas-Ferreira, P., Byrne, K., Praekelt, U. M., Meacock, P., Régnacq, M. \& Boucherie, H. (1994). Induction of major heat shock proteins of Saccharomyces cerevisiae, including plasma membrane Hsp30, by ethanol levels above a critical threshold. Microbiology 140, 3031-3038.

Piper, P. W., Ortiz-Calderon, C., Holyoak, C., Coote, P. \& Cole, M. (1997). Hsp30, the integral plasma membrane heat shock protein of yeast, is a stress-inducible regulator of plasma membrane $\mathrm{H}^{+}$ATPase. Cell Stress Chaperones 2, 12-24.

Piper, P. W., Mahé, Y., Thompson, S., Pandjaitan, R., Holyoak, C., Egner, R., Mühlbauer, M., Coote, P. \& Kuchler, K. (1998). The Pdr12 ATP-binding cassette (ABC) transporter is required for development of weak organic acid resistance in yeast. EMBO J $17,4257-4265$.

Régnacq, M. \& Boucherie, H. (1992). Isolation and sequence of HSP30, a yeast heat-shock gene coding for a hydrophobic membrane protein. Curr Genet 23, 435-442.

Riou, C., Nicaud, J. M., Barre, P. \& Gaillardin, C. (1997). Stationary- phase gene expression in Saccharomyces cerevisiae during wine fermentation. Yeast 13, 903-915.

Ruis, H. \& Schüller, C. (1995). Stress signalling in yeast. BioEssays 17, 959-965.

Schüller, C., Brewster, J. L., Alexander, M. R., Gustin, M. C. \& Ruis, H. (1994). The HOG pathway controls osmotic regulation of transcription via the stress response element (STRE) of the Saccharomyces cerevisiae CTT1 gene. EMBO J 13, 4382-4389.

Smith, B. J. \& Yaffe, M. P. (1991). Uncoupling thermotolerance from the induction of heat shock proteins. Proc Natl Acad Sci USA 88, 11091-11094.

Sorger, P. K. (1991). Heat shock factor and the heat shock response. Cell 65, 363-365.

Varela, J. C. S., Praekelt, U. M., Meacock, P. A., Planta, R. J. \& Mager, W. H. (1995). The Saccharomyces cerevisiae HSP12 gene is activated by the high osmolarity glycerol pathway and negatively regulated by protein kinase A. Mol Cell Biol 5, 6232-6245.

Watt, R. \& Piper, P. W. (1997). UBI4, the polyubiquitin gene of Saccharomyces cerevisiae, is a heat shock gene that is also subject to catabolite derepression control. Mol Gen Genet 253, 439-447.

Wenzel, T. J., Teunissen, A. W. R. H. \& Steensma, H. Y. (1995). PDA1 mRNA: a standard for quantitation of mRNA of Saccharomyces cerevisiae superior to ACT1 mRNA. Nucleic Acids Res 23, 883-884.

Winderickx, J., de Winde, J. H., Crauwels, M., Hino, A., Hohmann, S., Van Dijck, P. \& Thevelein, J. M. (1996). Expression regulation of genes encoding subunits of the trehalose synthase complex in Saccharomyces cerevisiae. Mol Gen Genet 252, 470-482.

Received 24 June 1998; revised 10 September 1998; accepted 30 September 1998. 